
\title{
25 Research Soure \\ Enhanced WSN Routing Protocol for Internet of Things to Process Multimedia Big Data
}

\author{
Vani Rajasekar \\ Kongu Engineering College \\ Premalatha Jayapaul \\ Kongu Engineering College \\ Sathya Krishnamoorthi \\ Kongu Engineering College \\ Muzafer Saracevic ( $\nabla$ muzafers@gmail.com ) \\ University of Novi Pazar https://orcid.org/0000-0003-2577-7927 \\ Mohamed Elhoseny \\ American University in the Emirates \\ Marwan Al-Akaidi \\ Mansoura University
}

\section{Research Article}

Keywords: nternet of Things, Multimedia, Big Data, WSN Routing Protocol

Posted Date: April 7th, 2021

DOl: https://doi.org/10.21203/rs.3.rs-368957/v1

License: (c) (1) This work is licensed under a Creative Commons Attribution 4.0 International License. Read Full License

Version of Record: A version of this preprint was published at Wireless Personal Communications on August 8th, 2021. See the published version at https://doi.org/10.1007/s11277-021-08760-1. 


\title{
Enhanced WSN Routing Protocol for Internet of Things to Process Multimedia Big Data
}

\author{
Vani Rajasekar ${ }^{1 *}$, Premalatha Jayapaul ${ }^{2}$, Sathya Krishnamoorthi ${ }^{3}$, Muzafer Saracevic ${ }^{4}$, \\ Mohamed Elhoseny ${ }^{5,6}$, Marwan Al-Akaidi ${ }^{6}$ \\ ${ }^{l}$ Dept of CSE, Kongu Engineering College, Perundurai, Erode, India, vanikecit.cse@kongu.ac.in \\ ${ }^{2}$ Dept of IT, Kongu Engineering College, Perundurai, Erode, India, jprem@kongu.ac.in \\ ${ }^{3}$ Dept of CT/UG, Kongu Engineering College, Perundurai, Erode, India, vanikecit.cse@kongu.edu \\ ${ }^{4}$ Dept of Computer Sciences, University of Novi Pazar, Serbia, muzafers@uninp.edu.rs \\ ${ }^{5}$ College of Computer Information Technology, American University in the Emirates, Dubai, United Arab \\ Emirates, melhoseny@ieee.org \\ ${ }^{6}$ Faculty of Computers and Information, Mansoura University, Egypt, marwan.alakaidi@aue.ae
}

\begin{abstract}
Due to the massive use of wireless Internet of Things (IoT), the advent of multimediabig-data (MBD) in recent decades poses numerous obstacles for successful contact with the virtual era. Mobile Adhoc Network-based IoT (MANETIoT) framework is increasingly common in this regard owing to its increased communication protocols and economic efficiency. MANET comprises arbitrary, battery-driven, roaming nodes that do not have an architecture that can handle the traffic and control the IoT network. In MANET-IoT, energy usage and traffic management for the handling of MBD information are significant issues. For rapid and precise response, it is essential to route or forward information like the locations of happenings and defected in a disaster. However, it is difficult to transfer this information to the Wireless Sensor Network in disaster areas because the current networks are a disaster that has been removed. In these situations, the transmission of opportunistic knowledge may play a vital role. Current opportunistic protocols need large messages for the restoration of the cluster that leads to more energy consumption and packet loss. To overcome these issues, this work proposes the reliable, energyefficient opportunistic protocol known as Density Clustering Opportunistic Routing Protocol (DCOR). This method sends information opportunistically in emergencies and disasters through a density-clustering protocol. Results from simulations demonstrate that the designed protocol exceeds several well-known current routing mechanisms for network energy usage and dissemination of information.
\end{abstract}

Keywords: Internet of Things, Multimedia, Big Data, WSN Routing Protocol.

\section{INTRODUCTION}

In today's world, the need for efficient knowledge sharing and control is critical in the rapid growth and extensive usage of IoT. Most significant is the management, due to the massive use of Mobile-IoT media networks and applications, of multimedia-big data (MBDs) in IoT [1]. Ensures and strategies for heterogeneous IoT communications and networks, therefore, need to be built. Mobile Adhoc Network-based IoT (MANET IoT) [2] is an evolving approach for IoT to tackle MBD with an effective IoT communication protocol. Sensor nodes exchange information through mobile multi-hop connections with one other on MANET-IoT scheme [3]. These networks do not 
have a predetermined network, such as Access Point (AP) or Base Station (BS), which implies that any node functions as a router to forward packets to other nodes. Clusters may have adjustable sources, like energy and memory, in an ad hoc network. The ad hoc routing protocol should be in line with nodes with higher levels of mobility which can alter network architecture. A straight routing protocol can provide many oversight details in a wide range of networks. In fact, with variable nodes with various resources, the functions within the node may vary to enable ad hoc network routing.

One solution to the above scenario is cluster-based routing. Cluster-based networking is one approach to have multiple nodes with varying resource levels. Cluster-based routing has been developed to minimize the number of routing details within the network. Clustering is intended to combine the sensor node into many clusters that enable the hierarchy of cross-clusters. This will improve current routes' life and thus reduce the downtime power. The portion of a cluster contains Cluster Head $(\mathrm{CH})$ for control of the cluster communication; Gateway Node (GN) for communicating data between groups; and Standard Nodes (SN) [4]. Standard nodes may either send packets to their head node or transfer them to GN. In conjunction, the overall interaction can be minimized by involving cluster head and gateway nodes in the routing of refresh messages that can be important if the volume of the network is high. Mobile ad-hoc network clusters have been extensively studied and the benefits of cluster analysis have been indicated by various techniques.

One potential methodology of hierarchical clustering, commonly used in wireless sensor networks (WSNs) [5] is Density Clustering Opportunistic Routing Protocol (DCOR). The purpose of this technique is to lower energy usage and prolong the network life through fixed node expectations. Opportunistic data sharing is used in the proposal DCOR protocol, which involves both a) density-dependent clustering and (b) isolated nodes. Each node would likely remain in cluster in a density-based cluster and share data between the cluster nodes. But on another view, isolated nodes (not in clusters) send network packets to a node between multiple nodes based on their likelihood of transmitting data. The Time to Return (TTR) factor amongst nodes is evaluated by clusters of this method. A node's power use varies widely depending on every cluster node's position. We conduct an experiment with a practical disaster zone model to test how well the opportunistic routing protocol is being suggested. The findings demonstrate that in opportunistic forwarding [6] or routing the suggested DCOR protocol improves network life and efficiency and decreases the packet loss ratio in comparison with state of art forwarding protocols and clustering protocols like LEACH, LORA, and TEEN.

The main contribution of this work is i) Incorporates edge computing with incident management opportunistic networking for MANET-IoT ii) Network detectors accumulate and dynamically analyze data to minimize the amount of information transferred iii) The sensors build clusters based on densities [7], where even the sensors represent cluster - head with the nearest nodes. This method decreases the number of data collectors and transceivers and similarly delivers energy across sensor nodes iv) The node which is not included in any group transfers information to the forwarder nodes opportunistically. These nodes reach the cluster that is nearest to them when moving $v$ ) if a node leaves the cluster due to network congestion, the process of restoring a cluster begins based on its property called Node's Minimum Connectivity (NMC) [8]. This property guarantees the energy and restoration of the clusters compared with the current cluster restore process. Protocols involving all the nodes after every round during cluster restore.

The remainder of the paper is organized as section 2 describes the related works on existing network protocols for MANET and Sensor Networks. Section 3 describes the principle of the proposed approach Density Clustering Opportunistic Routing Protocol (DCOR). Section 4 describes result analysis of the proposed approach and its comparison with existing routing protocol approaches. Section 5 depicts the conclusion of the proposed DCOR approach on multimedia big data and its future directions. 


\section{RELATED WORKS}

To monitor the effectiveness of the technique used and to verify its reliability in networking [9], routing protocols should be assessed. It is complicated and expensive to use an actual ad hoc network in an experiment, and such tests do not usually produce meaningful results. The actual network doesn't offer the convenience to vary the various environmental parameters and causes the problem of extracting results as well. This is because most of the performance evaluation work depends on the theory of simulation. The simulation makes it much easier under several conditions to evaluate the protocols.

The simulator, which is a device designed with a particular language, allows various environmental variables to be varied, including the increase of active units, the number of moving units, the speed of the movements, the network area, and the distribution of the units within that territory. Originally, each unit is positioned in the simulator space arbitrarily. Over a certain amount of time, a unit stays in its present position and then selects a new speed and a new place to which it travels. This same action is replicated by every group until around the end of this process. One of the major issues of sensor networks is energy efficiency [10]. As such, it is also very expensive and often difficult to recharge energy sources. Consequently, to operate, the sensors must conserve energy as much of it as feasible. Every sensor network performs an important role in the transfer of data because sensor networks perform in a hop-based routing mode [11]. Figure 1 shows the general structure of the wireless sensor network.

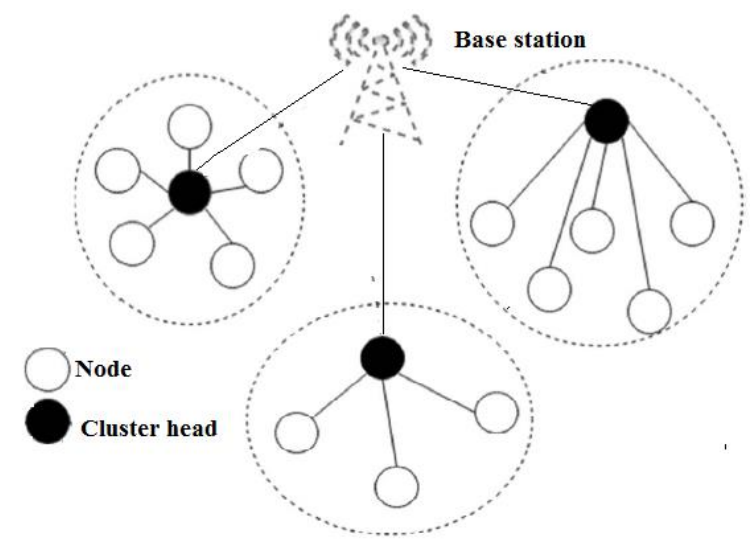

Figure 1. Structure of WSN

Traditional routing involves unicast traffic that transfers the packet from one node to another node in the hop-by-hop network. ExOR technique defined by IEEE 802.15 as a unicast routing scheme to improve bit rates in wireless multi-site networks [12]. It is worth noting that coding procedures is not introduced by ExOR, but it still enhances system performance.

The source node splits the data into groups (called generations or lots) through ExOR, and then notifies the packets by assigning a preference between the transmitters. If a packet is received by the highest priority transmitter, it waits for some time according to the priority and then for an ACK. Alternatively, packet recognition is the responsibility of a lower priority transmitter. A transmitter can, however, transmit even packets which are not recognized by higher-priority nodes.

\subsection{Analysis of existing protocols}

A very well recognized clustering protocol for wireless communications, the Low Energy Adaptive Clustering Protocol (LEACH) [13]. This protocol selects a random cluster head (CH) 
across all nodes based on a metric known as a $\mathrm{CH}$ likelihood. Used this Time Division Multiple access Scheme (TDMA) the membership of each node sends information to their $\mathrm{CH}$. All data, fine aggregate, are collected by the $\mathrm{CH}$ and transferred to the ground station for further analysis. In LEACH data is sent regularly from the cluster members and the cluster creation occurs after each round that is not energized. In Threshold Sensitive Energy Efficient Sensor Networks Protocol (TEEN) [14] a node can detect and transfer a value based on the least parameter rule system on the sensor (i.e. pressure, humidity). If the significance of the attribute increases by a certain threshold and the next time the node detects and communicates another outcome of interest, it is assigned to the node. Therefore in the TEEN Protocol, there are fewer amounts of sensed and broadcast cases.

The LEACH and TEEN protocols, however, might not be working well in emergencies and disasters. Another well-defined existing protocol is LORA (Load-balancing Opportunistic Routing for Asynchronous duty-cycled in sensor networks [15]. This method establishes candidate domains for all potential data forwarder candidates to prevent overflowing from source to a group of predefined forwarder nodes. The source node selects a candidate based on a metric determined using directional spread, selection of the transmission distance, perpendicular allocation, and the distribution of residual electricity. As well this method seems to be computationally costly and may not make it possible for clusters to acquire more power. Every node determines locally, utilizing greedy ways, about the forwarder node and generates lots of data to make such a determination. Epidemic routing protocols are used by cooperative communications in that node sends or forum messaging to all the other domains that generate a huge overhead communication ranking, too.

In contrast to conventional best approach routing, ExOR and MORE show that opportunistic routing [16] increases energy and throughput efficiency. These routing platforms are filtering mechanisms identified by the end-to-end redirector and offer priority to the transmitting applications using the destination sequence number. Each packet manager, on the other hand, separately decides its applicant along the routing path, known as the hop-by-hop redirection package. Another interesting opportunistic protocol is proposed by Zaho et al [17] called EnergyEfficient Opportunistic Routing Protocol (EEOR). This protocol seeks to decrease the node or the entire channel's energy usage, but the retained energy balance and the transmission delay from end to end are not taken into account. Throughout this procedure, the candidate's preference and prioritization program are optimized to minimize energy usage. In addition, EEOR considers the data transmission case to be adjustable. The EEOR takes less effort to send and receive data than that of the ExOR method, and the total EEOR forwarding list size is smaller than the EXOR.

EEOR performs better than EXOR throughout the event of total energy. EEOR performs better than ExOR by contrasting both protocols for packet size and end-to-end latency. Fradj et al [18] proposed another opportunistic protocol known as Range-based Opportunistic Protocol (ROR). The ROR protocols aim to decrease energy consumption, increase the life of the sensor array, increase the amount of transmitted data and eventually decrease the number of control packets. ROR's basic principle is to decrease the number of candidates selected to preserve the optimal energy for the networks. SOAR [19] is one of the opportunistic routing protocols based on the proactive link. This is a proactive routing protocol in which each asserts a routing table includes the target, the default route chosen, and the list of participating nodes. The steps involved in routing are as follows:

- Step 1: Each node transmits information about the effectiveness of the links regularly.

- Step 2: A node decides the predefined path and the collection of transition nodes that can be transmitted, based on this evidence.

- Step 3: Based on such knowledge, thus it transmits data packets.

- Step 4: The transfer category nodes store the packet and show a timer for the transmission. 
- Step 5: The node that is nearest to the target but has a tiny timer transfers the packet sooner.

- Step 6: To prevent dual transfers, the other nodes will delete the associated packet from their buffers.

\section{PROPOSED DENSITY CLUSTERING OPPORTUNISTIC ROUTING PROTOCOL}

This approach depends on Density Clustering Opportunistic Routing Protocol (DCOR). It will broadcast and forward the stored data to the nearest node. This allows the data collected to be forwarded or transmitted to the nearest node or nearest node to the endpoint, as the nodes are deployed and which lose network access. The flow of the proposed approach is given in Figure 2.

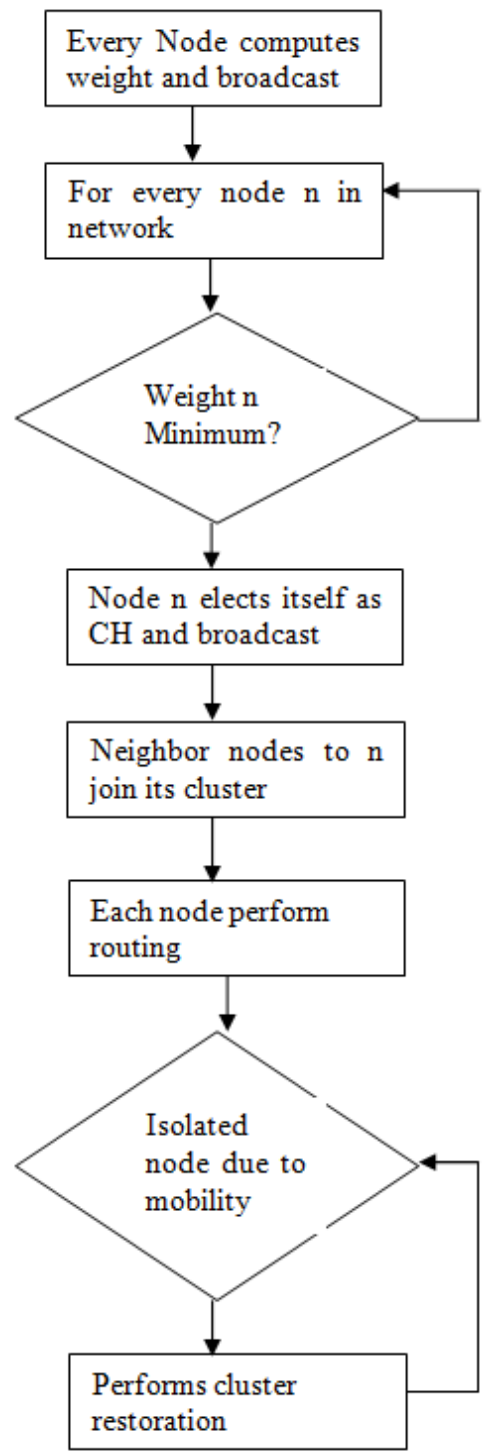

Figure 2. The flow of DCOR Protocol

\subsection{Parameters}

The Node's Minimum Connectivity (NMC) symbolizes the connectivity energy between two nodes as they are traveling. This property also reflects a node's efficient transmission of data packets. The NMC of two nodes $m$ and $n$ is denoted as 


$$
N M C_{m n}=\frac{R-D}{V_{m}+V_{n}}
$$

Where $R$ is the communication range between the node $m$ and $n, D$ is the distance between two nodes, $V_{m}$ and $V_{n}$ are the velocity of node $m$ and $n$.

The Global NMC (GNMC) is denoted as a network that is formed based on the nodes having the same GNMC between its nodes. Weight $W$ represents node is stable or non-stable. It is calculated as below. Number of neighbor nodes for $m$ is

$$
\begin{aligned}
& Y=\mid \text { neighbors }(m) \mid \\
& X=\sum_{n \in \text { neighbors }(m)} \frac{N M C_{m n}}{G N M C} \\
& \text { Weight }=X-Y
\end{aligned}
$$

\subsection{Cluster formation based on form}

Equation 3 denotes weight calculation for each node. If the weight of the node $n$ is equal to or closer to zero, then Node's Minimum Connectivity (NMC) of that particular node with all its neighbors is equal to Global NMC (GNMC). This implies that the node $n$ is stable and good to become cluster head $(\mathrm{CH})$. The following algorithm represents the selection of cluster heads.

\section{Algorithm 1: CH selection}

Input: A node $n$, GNMC, minimum nodes of the cluster

Output: Cluster Head (CH)

Process:

Identify neighbors of the node $n$ as $\mathrm{N} 1$.

Find the weight of node $n$ and other nodes based on equation (2)-(4)

If $|N 1| \geq$ minimum number of nodes then

Select $n$ as core points

If weight $(n) \leq$ weight $(y), \forall y \in N 1$

Select $n_{\text {as CH }}$

Minimum number of nodes $=|N 1|$

Endif

Else

Endif

Choose $n$ as border points

For every $y \in|N 1|$ do

If $y$ does not belong to a member of the cluster

If $N M C_{n, y} \geq G N M C$

Include y in the cluster $c l \leftarrow\{y\}$

Else

Endif

Set $y$ as noise to $c l$

Endif

Endif

Return Cluster Head

Repeat the above steps till all the required clusters are formed.

End 
The $\mathrm{CH}$ selection process denotes each node $n_{\text {computes its weight, identifies total neighbor nodes }}$ present, and broadcasts that information to all nodes. The nodes which contain the lowest weights are assumed to be $\mathrm{CH}$. All other nodes who have lower weight but not selected as $\mathrm{CH}$ are said to form a cluster.

\subsection{Routing technique}

As clusters are formed, nodes begin to gather information and thus data transfer or routing begins. If, due to movement recognized as a noise node, a node disconnected from a cluster, it is important to restore the cluster before it can send information.

Case 1: When a node $n$ has some information to relay, it passes data to its $\mathrm{CH}$ because it is a member of the cluster. If the node $n$ is disconnected, or a cluster boundary node, the connection is first attempted to restore and afterward data transferred. It also moves data to a nearby node on an opportunistic basis.

Case 2: When the node $n$ obtains the data information from various nodes, if the node $n$ is a part of the cluster then it interacts with $\mathrm{CH}$ and forwards the packets to $\mathrm{CH}$. And if node $n$ is a boundary node, it is indeed a noise that is either an element of a cluster or not the $\mathrm{CH}$ transfers data to the next hop to the endpoint. When a node $\mathrm{n}$ is a cluster member, the data is passed to the $\mathrm{CH}$. If $n$ is not part of a cluster, it needs to be within two adjacent Clusters' contact ranges. The node $\mathrm{n}$ then transmits a message that retrieves the nearest cluster and enters a cluster by preserving connection.

Case 3: The node could also lose communication with other nodes thus becoming disconnected. The topology of the disaster network is given in Figure 3.

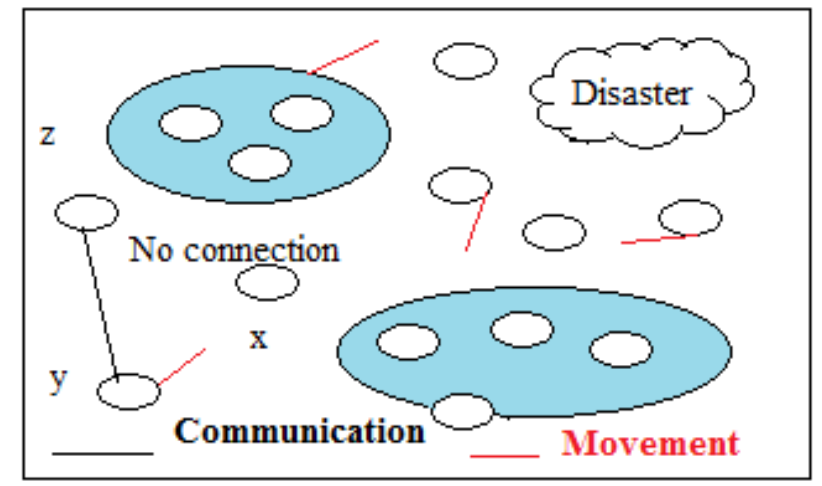

Figure 3. Topology of Disaster Network

The diagrammatic representation of opportunistic forwarding is given in Figure 4. The clusterbased transmission is specified in Figure 5.

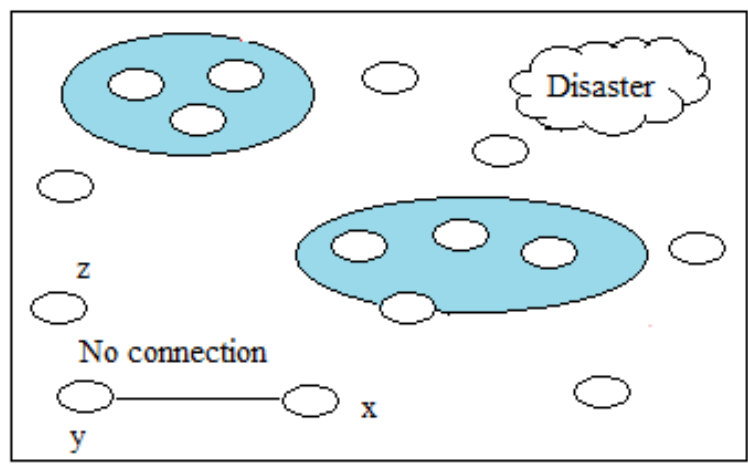

Figure 4. Message Forwarding Using Opportunistic 
In these, node $\mathrm{z}$ does not belong to the cluster hence when it receives data or senses any event it will forward that to the nearest neighbor. The message is now forwarded to node y. But y is also disconnected but when it mobiles it will reach the cluster region and able to transfer the data to node $\mathrm{x}$. The node $\mathrm{x}$ forwards the data to the nearest neighbor in the cluster thereby transfers the data to the destination (see Figure 5).

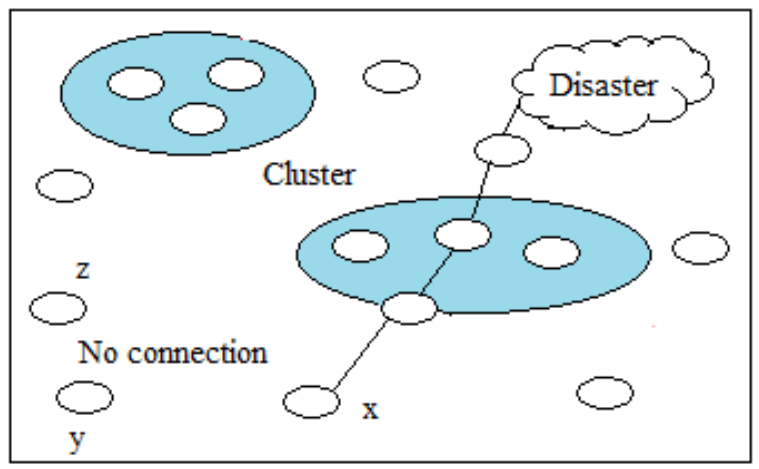

Figure 5. Transmission Based On CLuster

\subsection{Selection of Forwarding node}

The forward node needs to be selected during routing. To do this every node retains the $N M C$ value against its subsequent nodes and transfers information towards the next node having maximum $N M C$. Since the proposed protocol refers to disaster conditions in which every node moves to the emergency or disaster at about the same pace, where it continues to interact with a certain number of nodes. Therefore the source node which activates the data transfer can be enabled, through contact with these nodes, to transfer information to the receiver.

\subsection{Restoration of Clusters}

Because nodes in opportunistic networks are much more likely roaming, they enter or are disconnected at the cluster frontier. The restoration of the cluster is therefore necessary. The $x$ boundary node begins the restoration of the cluster by choosing the best nominee for the preservation of the relation from their noisy neighbors. This is represented in Figure 6 and Figure 7 at two conditions when the node $x$ is not a part of the cluster and when the node $x$ is the cluster's border node.

Case 1: When a node $x$ is the border node of the cluster, this should execute the restoration of the clustering algorithm. The Global $N M C$ referred as GNMC should be maintained by the node with maximum communication range as $R$ of node $y$ is given below.

$$
N M C_{y, x}=G N M C=\frac{R-D}{V_{x}+V_{y}}
$$

Where $V_{x} V_{y}$ denotes the border node's velocity and $D$ refers the distance among two nodes $x$ and $y . R$ implies the range of communication of all the nodes in the cluster. The above equation (5) can be used to increase the restoration of connectivity. This is represented in Figure 6. 


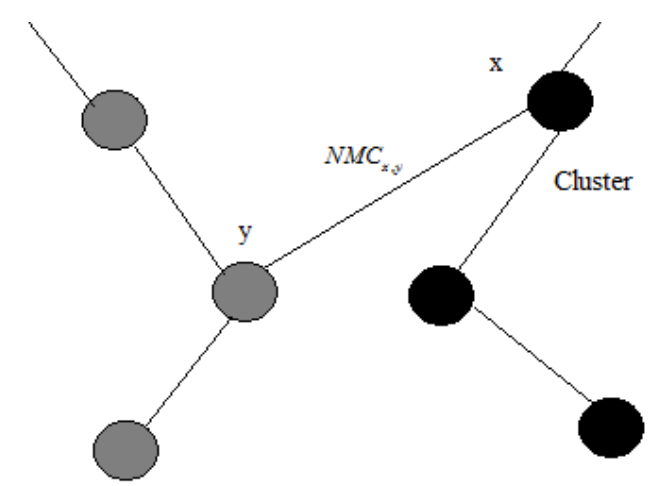

Figure 6. When the node $x$ is the border node of Cluster

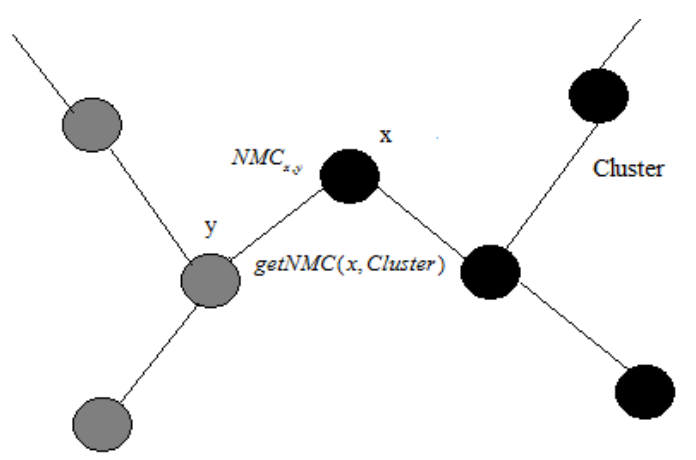

Figure 7. When the node $x$ is not a part of Cluster

Case 2: When a node $x$ is isolated, the protocol identifies the minimum $N M C$ between $N M C_{y, x}$ and $N M C_{C l u s t e r, x}$. The range $R$ has to be increased to maintain GNMC. This scenario is depicted in Figure 7. Here the node $x$ is referred to as noise, $x \notin$ Cluster and it is in the sensing range of all its neighbors. The boundary nodes from one of the clusters start the restoration process on clusters to repair the connections. The boundary node is usually enabled for the cluster that has a $\mathrm{CH}$ minimum weight to link to the other cluster. The steps involved in cluster rest. The oration is given in algorithm 2.

\section{Algorithm 2: Cluster Restoration}

Input: A noden, GNMC, cluster's minimum node

Output: Cluster Head $(\mathrm{CH})$

Process:

Identify $x$ where $N M C_{y, x}=\max$ imum in which $x$ consider being noise

Compute $x=\operatorname{Identify\_ Best}(y, x)$ were $N M C_{y, x}=\max$ imum and $x \in$ noise

If a node $x$ is member (Cluster) then

Increase $R$ of $y(\operatorname{getNMC}(x, y), G N M C)$

Else

temp $=\min (\operatorname{getNMC}(x, y)$, getNMC $(x$, Cluster $))$

Increase $R$ of $y($ temp, GNMC)

Endif

End 


\section{RESULTS AND DISCUSSIONS}

The performance analysis and results of the proposed enhanced DCOR protocol are described in the following section.

\subsection{Experimental setup}

The proposed DCOR protocol is simulated in NS-3 [20]. The simulation result witnessed that the proposed approach is better in performance when compared to LEACH, LORA, and TEEN protocol. This section describes the network model, energy model, mobility model of the proposed approach. The simulation parameters are listed in Table 1.

Table1. Simulation parameter of the proposed approach

\begin{tabular}{|l|l|}
\hline \multicolumn{1}{|c|}{ Parameters Used } & \multicolumn{1}{c|}{ Value } \\
\hline Simulation area & $2000 \times 2000 \mathrm{~m}^{2}$ \\
\hline Total number of nodes & 100 \\
\hline Communication range & $200 \mathrm{~m}$ \\
\hline Protocol for transport & $\mathrm{UDP}$ \\
\hline MAC Protocol & IEEE 802.11 \\
\hline Speed of mobility & $5 \mathrm{~m} / \mathrm{s}$ \\
\hline Model of mobility & Random \\
\hline Each round's duration & $200 \mathrm{~s}$ \\
\hline Type of bit rate & $\mathrm{CBR}(\mathrm{Constant}$ Bit Rate $)$ \\
\hline Size of the data packet & $1 \mathrm{~kb}$ OR 128 bytes \\
\hline The transmission rate of data & $250 \mathrm{kbits} / \mathrm{s}$ \\
\hline
\end{tabular}

\subsubsection{Sensor network model}

The proposed sensor network model consists of $N$ nodes denoted by Graph $S(E, V)$ where $E$ denotes a set of edges and $V$ denotes a set of vertices. Consider two nodes $\mathrm{x}$ and y communicate with each other in the communication range $R$. The network of the proposed approach is sensor-based opportunistic double range property [21] denoted with $R=N \times S$ where $N \geq 2$ and $S$ denotes sensing range. We allow a network of $4.0 \mathrm{~km} 2$ (about $2 \mathrm{kmX} 2 \mathrm{~km}$ ), as the disaster areas around which rescue personnel have to be linked to restoration activities are a genuine area. Figure out that, given the scenario in which rescue crews are roaming slowly or moving in a particular part of the region, the pace of mobilization of rescue workers is expected about $5 \mathrm{~m} / \mathrm{s}$ or $300 \mathrm{~m} / \mathrm{min}$.

\subsubsection{Mobility Model}

BonnMotion [22] mobility generation generates random movement of the nodes, which randomly brings every node into the simulation range from 2000 X 2000 square meters. The sequence of mobility generation is Random Possibility (RP) which is provided by BonnMotion. The mobility patterns produced pauses for a certain time each node at its actual position before moving at a predetermined rate to some other completely random spot. In low frequency and controlled sensors, the IEEE 802.15.4 basic Zigbee approach is used. The same sensors are considered here for performing the simulation. 


\subsubsection{Energy Model}

When data is transmitted between sender and receiver certain energy is consumed for the nodes. The energy consumption model of the proposed approach is specified below. Energy consumption to transfer the data packet of size $N$ over the distance $K$ is specified as

$$
E_{T X}=N * E_{\text {elec }}+N * E_{f s} * K^{\alpha}
$$

Similarly, Energy consumption to receive the data packet is

$$
E_{R X}=N * E_{\text {elec }}
$$

Where

$E_{\text {elec }}$ is energy dissipated in transmitter electronic circuit

$E_{f s}$ is energy dissipated in RF amplifiers

The variable (exponent of path loss) is defined according to the surroundings. Excluding obstruction to the line of view, for free space $=2$. This energy storage system shows that the energy usage of a device is directly related to the data apparent size traveled by a data packet.

\subsection{Efficiency analysis on Energy}

In this section, the energy of the proposed approach is compared against well-known TEEN, LORA, and LEACH protocols. The cluster forming process starts after several cycles in LEACH and TEEN protocols in which all nodes take part in restore or update. And from the other hand, only if a node has become a boundary node or becomes disconnected the cluster reconstruction is started in this DCOR protocol. Furthermore, this cluster restoration process involves only boundary nodes and two nearby CHs. The remaining nodes stayed in their respective clusters and are not involved in the process of clustering. Consequently, in comparison to the current protocols of the LEACH and TEEN, the proposed DCOR is still more energy-saving and lesser packet drop ratio, and higher efficiency.

The parameters to be considered for this analysis are: $\mathrm{N}$ - number of nodes, $\mathrm{C}$ - number of clusters:

$$
\text { Average nodes }=\frac{N}{C}
$$

Total messages to be transmitted and received $=M$. Average message transferred for the formation of cluster $=N \times M(7)$

Restoration of Cluster in DOCR Protocol: Assume the number of nodes involved in cluster restoration is 3 where $3<N$. The average number of message in the proposed DCOR protocol in cluster restoration $=3 \times M(8)$. The order of cluster restoration is $O(n)$. On comparing (7) and (8), it is confirmed that the average transmission of a message in the proposed DCOR protocol is less than the existing TEEN and LEACH protocol. The time complexity of the existing LORA protocol is about $O\left(n^{2}\right)$ which is computationally high compared to the existing DCOR protocol. Since sensor network, energy demand is directly proportional to the number of messages sent the DCOR model energy consumption is projected to be much smaller than that of LEACH, TEEN, and LORA protocols. Likewise, as there are far more messages transmitted. The risk of loss of packets would be greater than those of the DCOR protocol in LEACH, TEEN, and LORA protocols. 


\subsection{Performance analysis}

The performance parameters to be considered for the proposed DCOR protocol is given below

- Energy: Complete energy absorbed over a channel by sensor nodes in the number of runs

- Throughput: The quantity of data transferred by a node per every second. The unit is considered as Gigabytes/second

- Packet Delivery Ratio: This is the probability of the total number of packets delivered to the destination to the total packets involved in the transmission

- End to End Delay: Delay includes the time needed for a full message between time to time from the origin to reach its destination. It reduces the network's efficiency.

Table 2 represents the various performance metrics such as throughput, packet delivery ratio, energy, and an end-to-end delay of the proposed DCOR protocol. The proposed approach is run for every 100 nodes.

Table 2. Performance analysis of the proposed approach

\begin{tabular}{|c|c|c|c|c|c|c|}
\hline S.No & $\begin{array}{l}\text { Performance } \\
\text { metrics }\end{array}$ & $\begin{array}{l}\text { Number of } \\
\text { Nodes }\end{array}$ & LEACH & TEEN & LORA & $\begin{array}{l}\text { Proposed } \\
\text { DCOR }\end{array}$ \\
\hline \multirow{4}{*}{1} & \multirow{4}{*}{$\begin{array}{l}\text { Energy } \\
\text { (Watts) }\end{array}$} & 25 & 32.554 & 31.25 & 37.650 & 25.18 \\
\hline & & 50 & 26.625 & 26.560 & 41.69 & 19.225 \\
\hline & & 75 & 22.736 & 23.562 & 29.35 & 15.25 \\
\hline & & 100 & 18.873 & 19.736 & 19.112 & 11.25 \\
\hline \multirow{4}{*}{2} & \multirow{4}{*}{$\begin{array}{c}\text { Throughpu } \\
\text { t } \\
\text { (bps) }\end{array}$} & 25 & 21578 & 23252 & 22561 & 24648 \\
\hline & & 50 & 23562 & 26786 & 25531 & 26361 \\
\hline & & 75 & 23896 & 28664 & 24650 & 28982 \\
\hline & & 100 & 24582 & 31765 & 28638 & 32237 \\
\hline \multirow{4}{*}{3} & \multirow{4}{*}{$\begin{array}{c}\text { Packet } \\
\text { Delivery } \\
\text { Ratio (\%) }\end{array}$} & 25 & 81.23 & 86.36 & 86.76 & 91.16 \\
\hline & & 50 & 82.29 & 83.45 & 87.12 & 90.12 \\
\hline & & 75 & 81.56 & 85.78 & 84.36 & 87.33 \\
\hline & & 100 & 81.13 & 84.71 & 83.12 & 88.26 \\
\hline \multirow[t]{4}{*}{4} & \multirow{4}{*}{$\begin{array}{l}\text { End-to- } \\
\text { End } \\
\text { Delay(sec) }\end{array}$} & 25 & 0.428 & 0.386 & 0.361 & 0.201 \\
\hline & & 50 & 0.500 & 0.398 & 0.378 & 0.292 \\
\hline & & 75 & 0.576 & 0.378 & 0.436 & 0.313 \\
\hline & & 100 & 0.592 & 0.435 & 0.486 & 0.323 \\
\hline
\end{tabular}

Figure 8 represents the energy consumption of the proposed approach. From the analysis, it is proved that the proposed approach has lower energy consumption compared to existing LORA, LEACH, and TEEN protocols.

Energy analysis

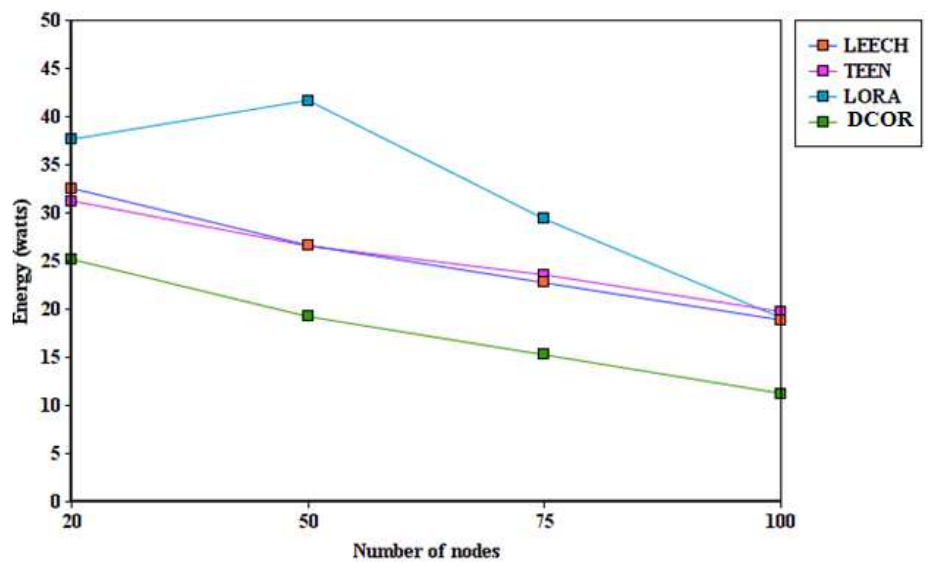

Figure 8. Energy Analysis of Proposed Approach 
The energy of DCOR protocol is calculated using the equation 6 and 7. The energy consumption on transmitting a message to destination in LORA protocol is $O\left(n^{2}\right)$ and the energy consumption of proposed DCOR is about $O(n)$. Figure 9 illustrates the throughput analysis of the proposed approach with existing protocols. The throughput of this scheme DCOR is greatly increased when compared to LEACH, LORA, and TEEN approach. This is due to the suggested ODCR protocol implements cluster recovery and easily generates clusters based on the worst network movements' situation. The existing approaches face serious connectivity loss that results in lower throughput which is not the scenario in DCOR.

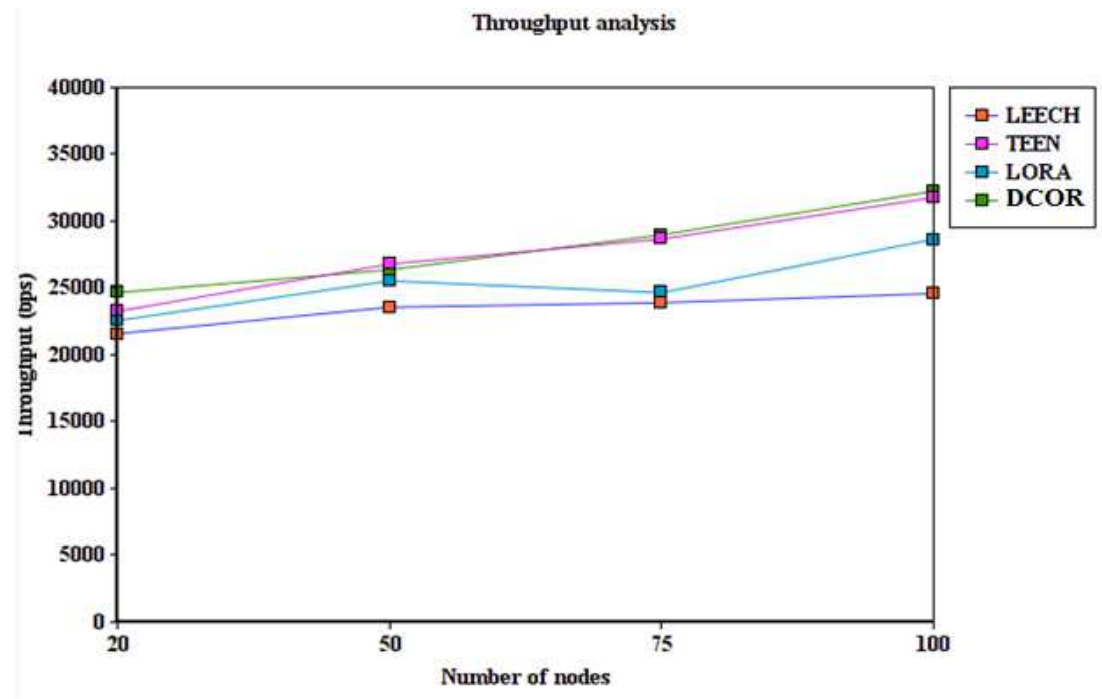

Figure 9. Throughput Analysis of DCOR

Figure 10 demonstrates the packet delivery ratio of the proposed DCOR. The result analysis denotes that the proposed approach has 50\% greater packet delivery ratio comparing with LEACH protocol. Also, it is $20 \%$ more packet delivery ratio compared to LORA protocol.

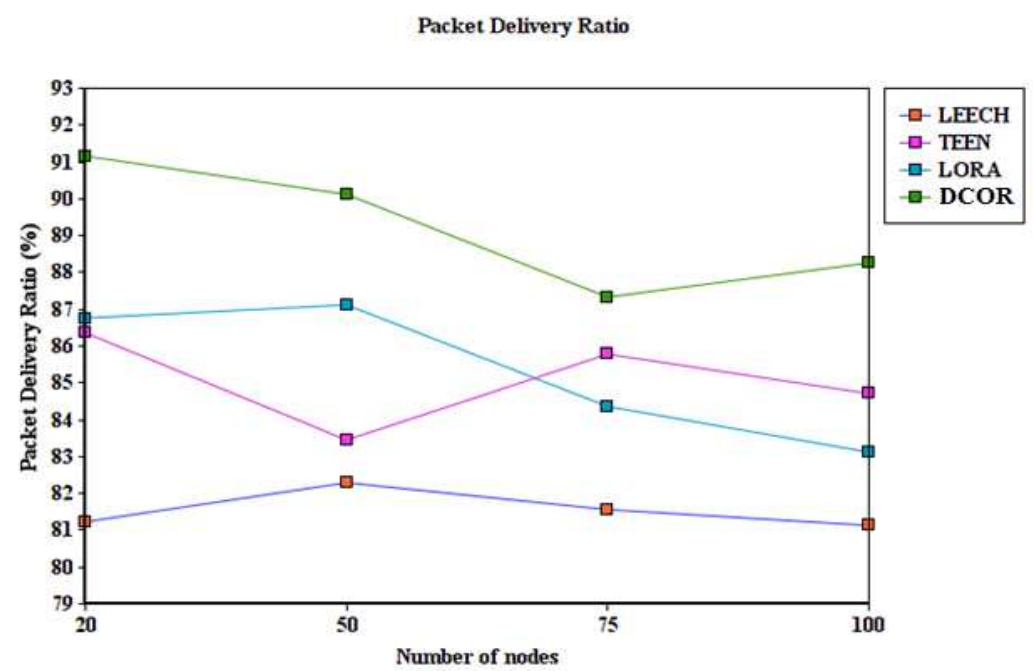

Figure 10. Packet Delivery Ratio of DCOR

Figure 11 represents the End to end delay of the proposed DCOR protocol. End-to-end delay reveals transmission delay from origin to destination. This delay indicates delays in the propagation 
and latency of each network intermediate node. To analyze the effect of delay in nodes on transmission the DCOR protocol is proposed.

From the result it is implicit that DCOR has much lower end-to-end delay compared to LEACH, TEEN and LORA approaches. The DCOR has 50\% lower delay compared to LEECH, LORA, and TEEN on approximation.

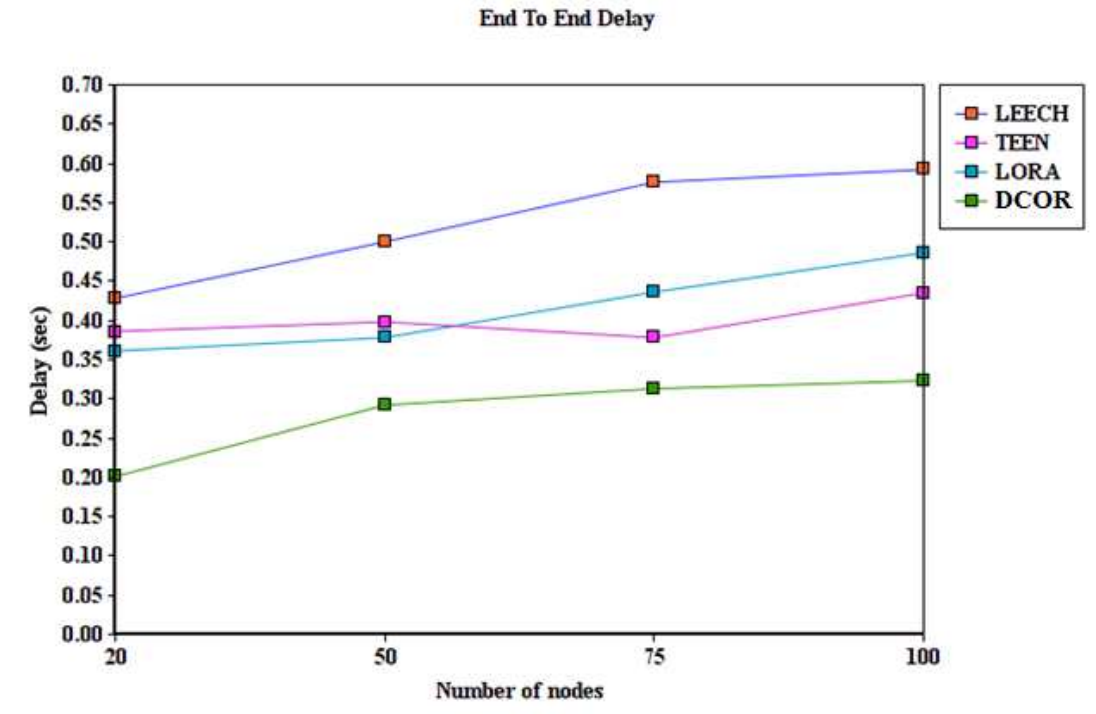

Figure 11. End to End Delay of DCOR

\section{CONCLUSION}

The scheme illustrated in this paper denotes an energy-efficient, dynamic, and Density Clustering Opportunistic Routing Protocol (DCOR) for MANETs. Regular nodes in WSNs transmit messages to cluster heads [23] and this data is collected periodically by the base station. To achieve this proposed protocol is mainly designed to transmit data and information sensor nodes and varied wireless network nodes in emergency and disaster conditions. In this approach, every node connects to a sensor network based on spatial clusters and shares information on certain cluster nodes, or forwards data to each node whenever a node with such a high likelihood of data being sent to the destination is sometimes encountered [24].

The performance of the proposed DOCR approach is evaluated in terms of Throughput, packet delivery ratio, end-to-end delay, and energy calculation. The DOCR protocol is compared with other existing approaches such as LORA, TEEN, and LEACHES concerning discussed performance metrics. Result analysis witnessed that the proposed DOCR routing protocol provides enhanced results compared to LORA, LEACH and TEEN approaches. In the future, the performance of the proposed approach is compared against localization error, latency, and other performance metrics. There is a plan to incorporate a reinforcement learning approach in DOCR protocol to achieve higher sensor efficiency.

Funding: This research received no external funding.

Conflicts of Interest: The authors declare no conflict of interest.

Availability of data and material (data transparency): Not applicable.

Author Contributions: Formal analysis, Methodology (V.Rajasekar, P.Jayapaul, S.Krishnamoorthi); Resources, Investigation (M.Saracevic); Conceptualization (M.Elhoseny, M.Al-Akaidi) 


\section{REFERENCES}

[1] I. Alameri, "MANETS and Internet of Things: the development of a data routing algorithm," Engineering, Technology \& Applied Science Research, vol. 8, pp. 2604-2608, 2018.

[2] R. Bruzgiene, L. Narbutaite, and T. Adomkus, "MANET network in internet of things system," Ad Hoc Networks, pp. 89-114, 2017.

[3] B. H. Al-Qarni, A. Almogren, and M. M. Hassan, "An efficient networking protocol for internet of things to handle multimedia big data," Multimedia Tools and Applications, vol. 78, pp. 30039-30056, 2019.

[4] F. Javed, M. K. Afzal, M. Sharif, and B.-S. Kim, "Internet of Things (IoT) operating systems support, networking technologies, applications, and challenges: A comparative review," IEEE Communications Surveys \& Tutorials, vol. 20, pp. 2062-2100, 2018.

[5] V. Rajasekar, K. Sathya, and J. Premalatha, "Energy Efficient Cluster Formation in Wireless Sensor Networks Based on Multi Objective Bat Algorithm," in 2018 International Conference on Intelligent Computing and Communication for Smart World (I2C2SW), 2018, pp. 116-120.

[6] M. S. Al-kahtani, L. Karim, and N. Khan, "Efficient Opportunistic Routing Protocol for Sensor Network in Emergency Applications," Electronics, vol. 9, p. 455, 2020.

[7] R. Borawake-Satao and R. Prasad, "Mobility aware multi-objective routing in wireless multimedia sensor network," Multimedia Tools and Applications, vol. 78, pp. 32659-32677, 2019.

[8] M. Saračević, Š. Plojović, and S. Bušatlić, "IoT Application for Smart Cities Data Storage and Processing Based on Triangulation Method," in Internet of Things (IoT), ed: Springer, 2020, pp. 317-334.

[9] V. Rajasekar, J. Premalatha, and K. Sathya, "Multi-factor signcryption scheme for secure authentication using hyper elliptic curve cryptography and bio-hash function," Bulletin of the Polish Academy of Sciences. Technical Sciences, vol. 68, 2020.

[10] M. H. Saračević, S. Z. Adamović, V. A. Miškovic, M. Elhoseny, N. D. Maček, M. M. Selim, et al., "Data Encryption for Internet of Things Applications Based on Catalan Objects and Two Combinatorial Structures," IEEE Transactions on Reliability, 2020.

[11] S. Velliangiri, S. A. Kumar, and P. Karthikeyan, Internet of Things: Integration and Security Challenges: CRC Press, 2020.

[12] V. Rajasekar, J. Premalatha, and K. Sathya, "Enhanced Biometric Recognition for Secure Authentication Using Iris Preprocessing and Hyperelliptic Curve Cryptography," Wireless Communications and Mobile Computing, vol. 2020, 2020.

[13] P. Marappan and P. Rodrigues, "An energy-efficient routing protocol for correlated data using CL-LEACH in WSN," Wireless Networks, vol. 22, pp. 1415-1423, 2016.

[14] R. Bria, A. Wahab, and M. Alaydrus, "Energy Efficiency Analysis of TEEN Routing Protocol with Isolated Nodes," in 2019 Fourth International Conference on Informatics and Computing (ICIC), 2019, pp. 1-5.

[15] A. Hawbani, X. Wang, Y. Sharabi, A. Ghannami, H. Kuhlani, and S. Karmoshi, "LORA: load-balanced opportunistic routing for asynchronous duty-cycled WSN," IEEE Transactions on Mobile Computing, vol. 18, pp. 1601-1615, 2018.

[16] Y. He, X. Tang, R. Zhang, X. Du, D. Zhou, and M. Guizani, "A Course-Aware Opportunistic Routing Protocol for FANETs," IEEE Access, vol. 7, pp. 144303-144312, 2019.

[17] M. Zhao, A. Kumar, P. H. J. Chong, and R. Lu, "A reliable and energy-efficient opportunistic routing protocol for dense lossy networks," IEEE Wireless Communications Letters, vol. 6, pp. 26-29, 2016. 
[18] H. B. Fradj, R. Anane, and R. Bouallegue, "Opportunistic routing protocols in wireless sensor networks," Wireless Personal Communications, vol. 104, pp. 921-933, 2019.

[19] M. Salehi, A. Boukerche, and A. Darehshoorzadeh, "Modeling and performance evaluation of security attacks on opportunistic routing protocols for multihop wireless networks," Ad Hoc Networks, vol. 50, pp. 88-101, 2016.

[20] Y. Mai, Y. Bai, and N. Wang, "Performance comparison and evaluation of the routing protocols for MANETs using NS3," 2017.

[21] S. T. Hasson and H. Abd Al-kadhum, "Developed clustering approaches to enhance the data transmissions in WSNs," in 2017 International Conference on Current Research in Computer Science and Information Technology (ICCIT), 2017, pp. 99-106.

[22] R. Vijayashree and C. Suresh Ghana Dhas, "Energy efficient data collection with multiple mobile sink using artificial bee colony algorithm in large-scale WSN," Automatika, vol. 60, pp. 555-563, 2019.

[23] O. Said, Y. Albagory, M. Nofal, and F. Al Raddady, "IoT-RTP and IoT-RTCP: Adaptive protocols for multimedia transmission over Internet of Things environments," IEEE access, vol. 5, pp. 16757-16773, 2017.

[24] V. Rajasekar, P. Jayapaul, and S. Krishnamoorthi, "Cryptanalysis and enhancement of multi factor remote user authentication scheme based on signcryption," Advances in Mathematics of Communications, p. 0, 2019. 
Figures

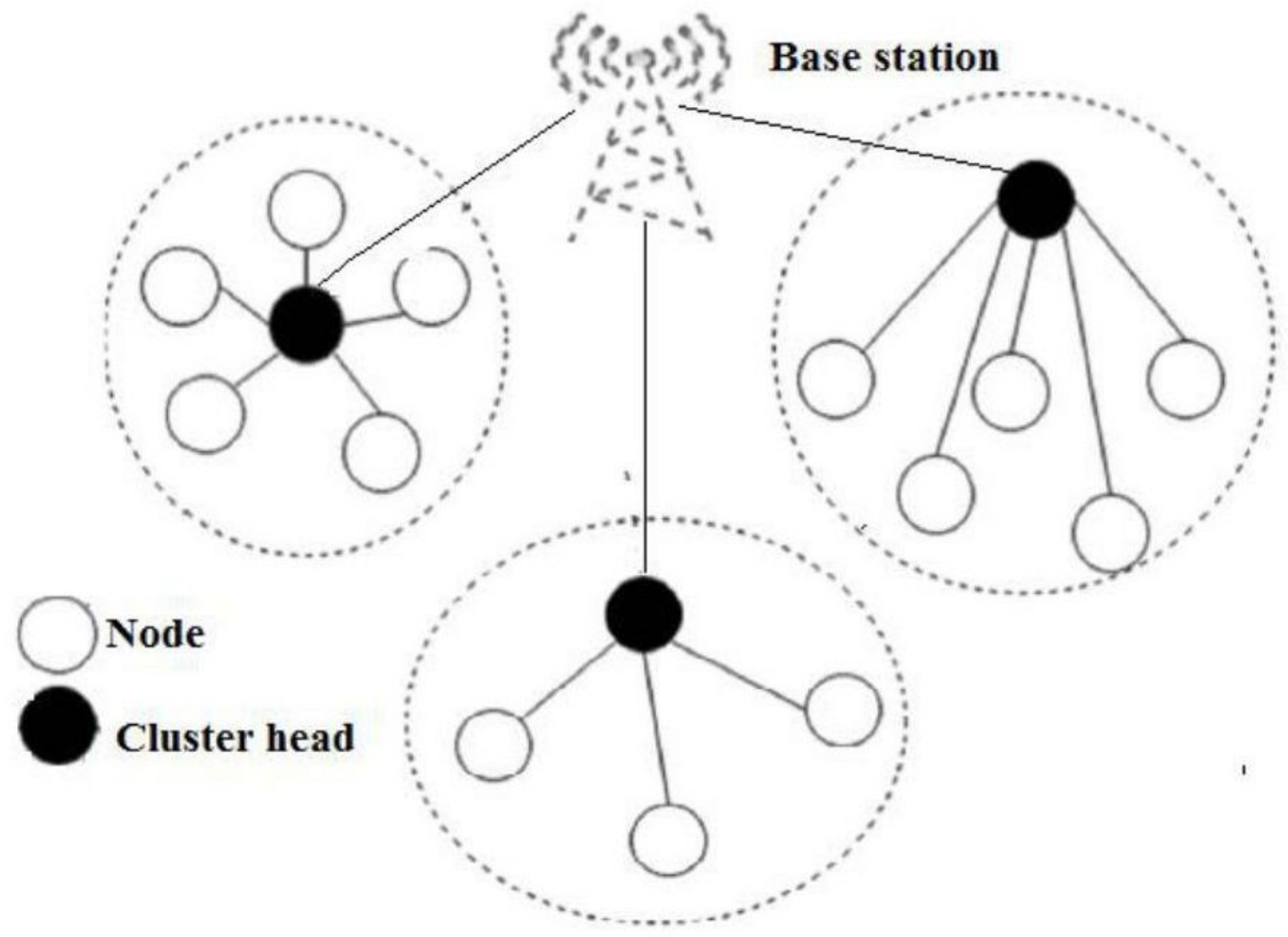

Figure 1

Structure of WSN 


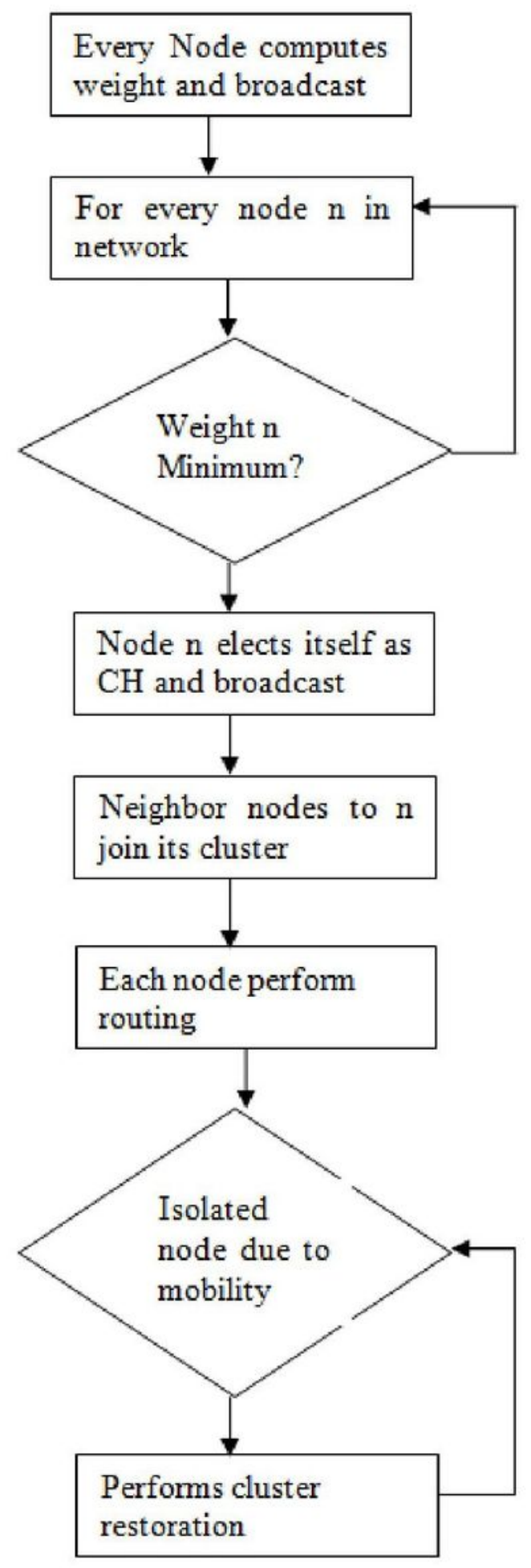

\section{Figure 2}

The flow of DCOR Protocol 


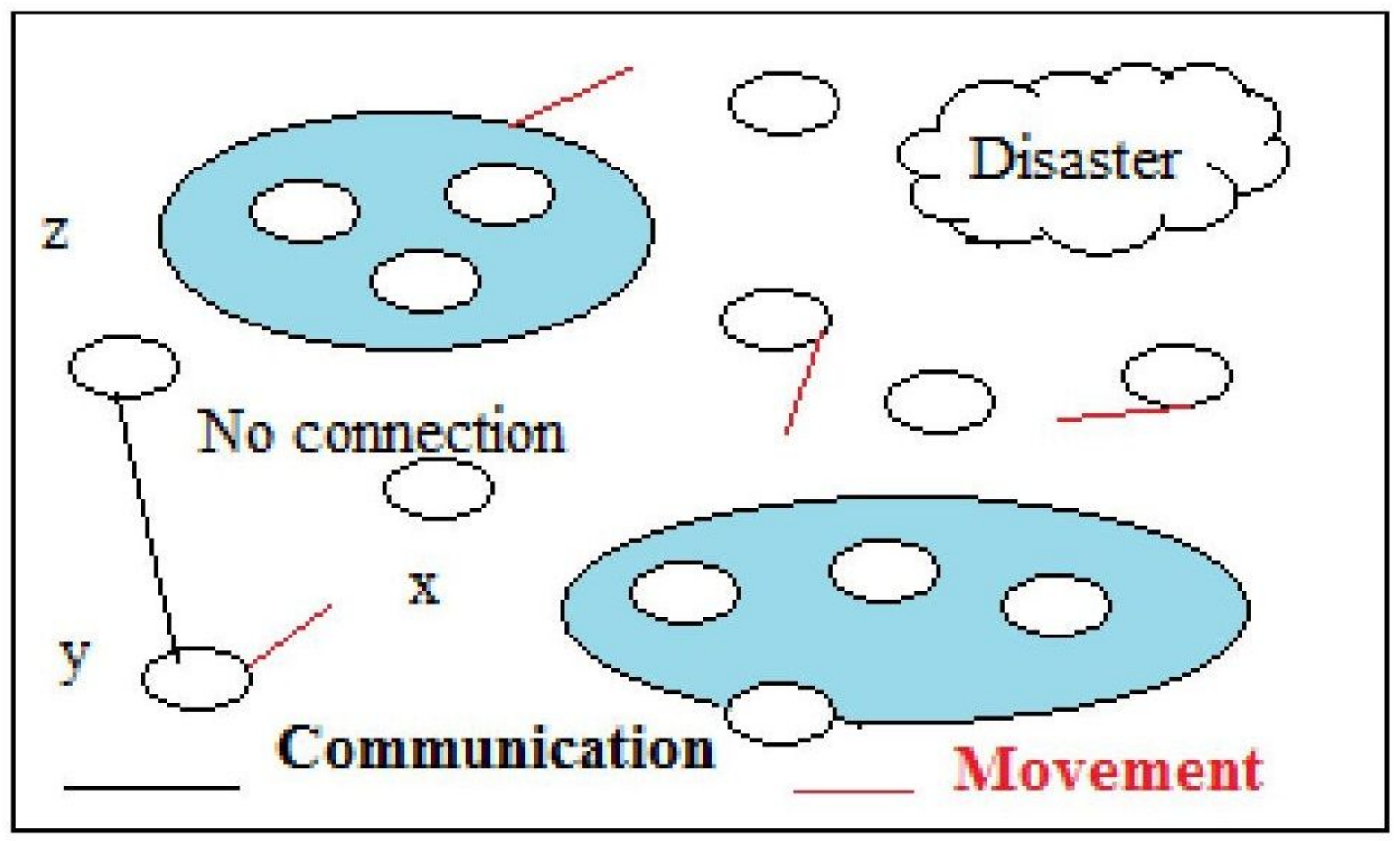

Figure 3

Topology of Disaster Network 


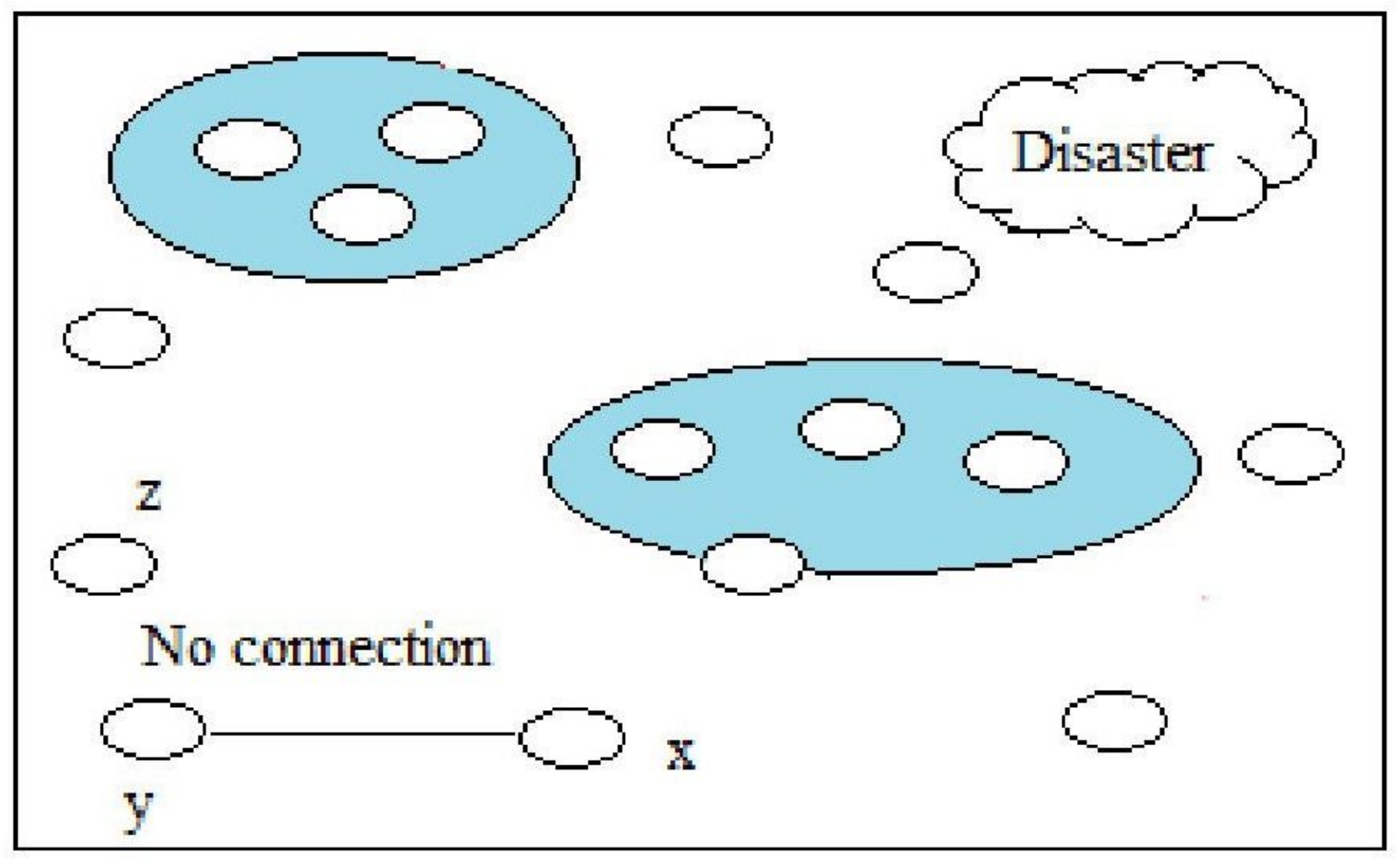

Figure 4

Message Forwarding Using Opportunistic 


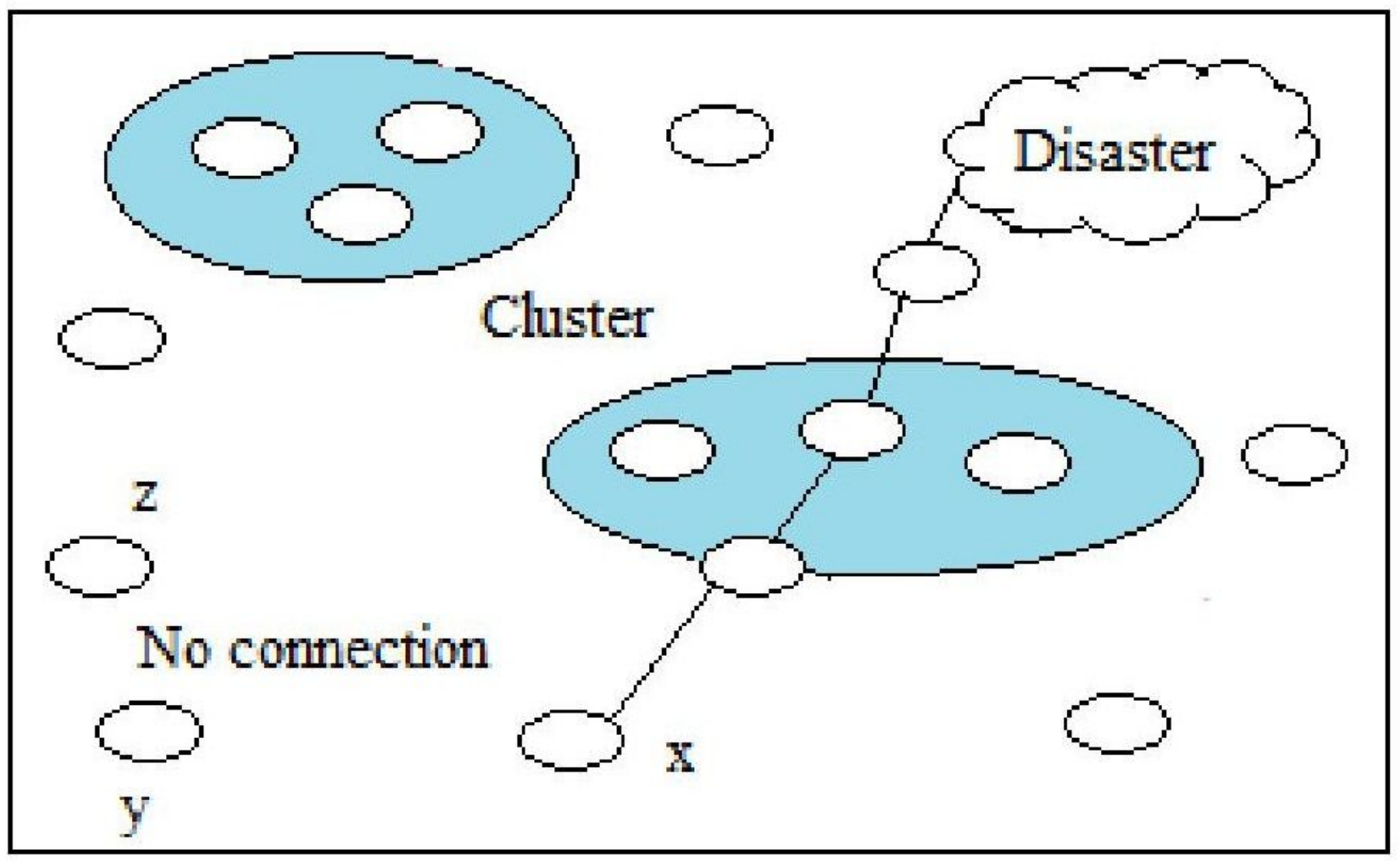

Figure 5

Transmission Based On CLuster 


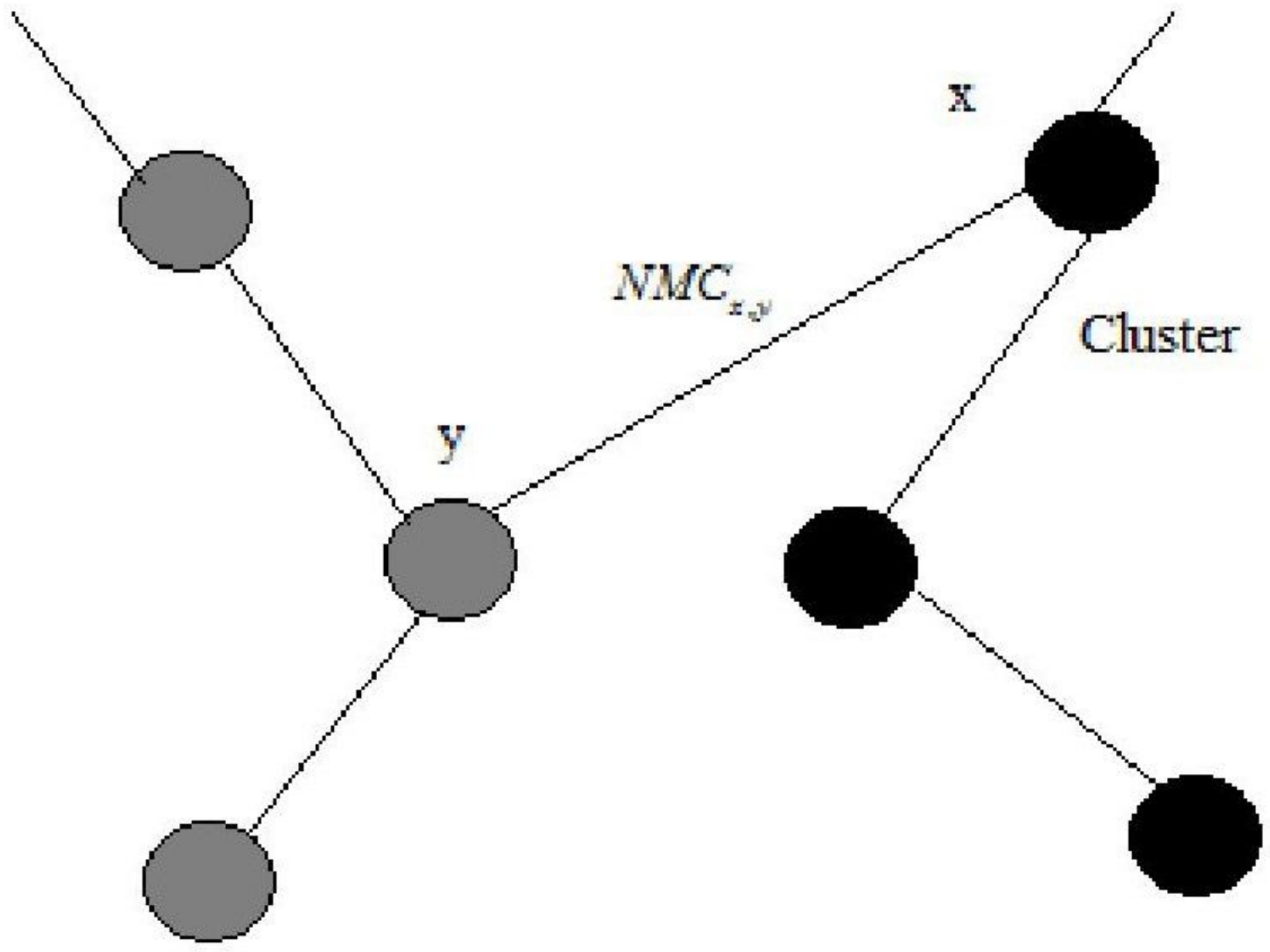

Figure 6

When the node $\mathrm{x}$ is the border node of Cluster 


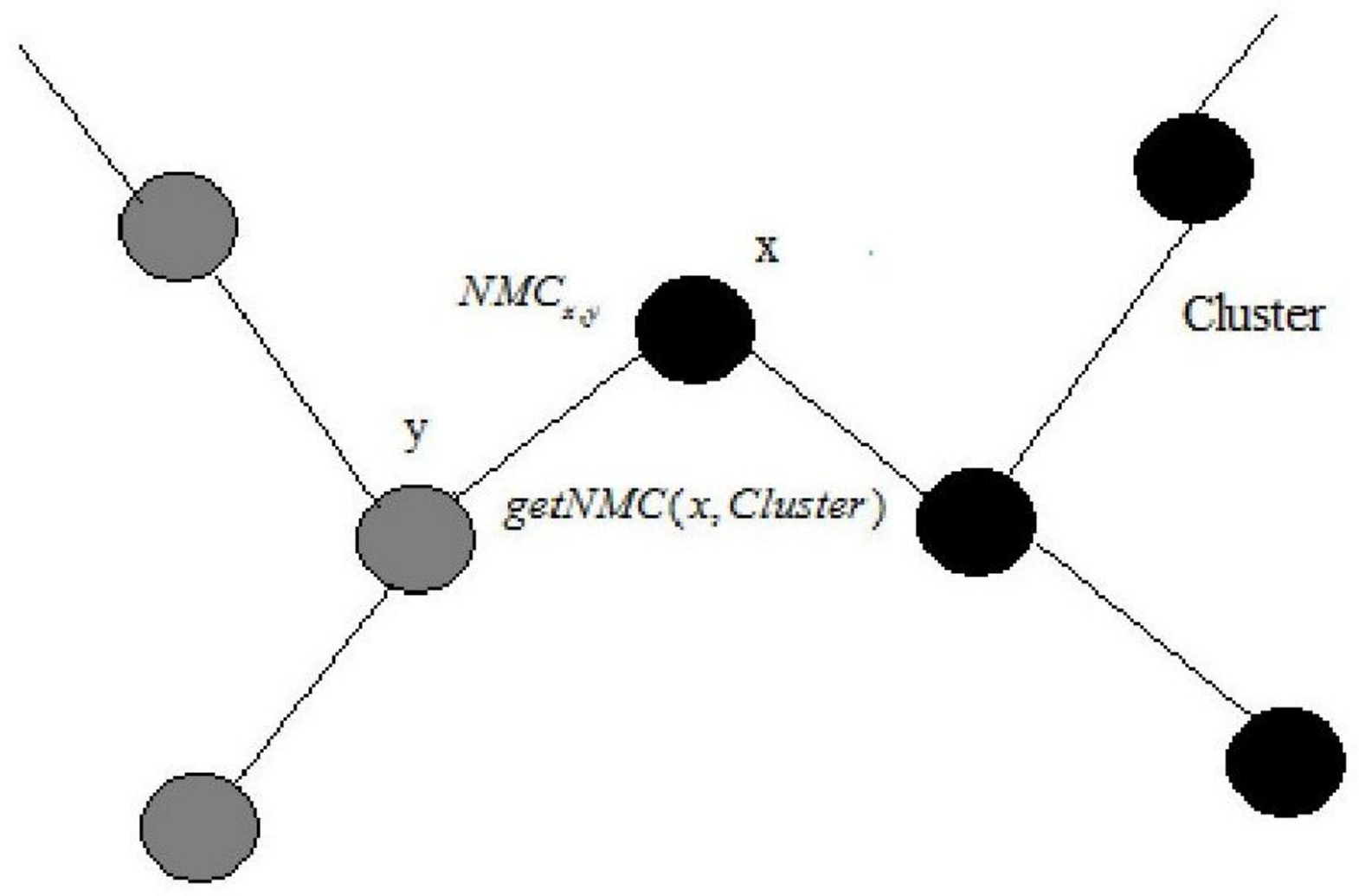

Figure 7

When the node $\mathrm{x}$ is not a part of Cluster 
Energy analysis

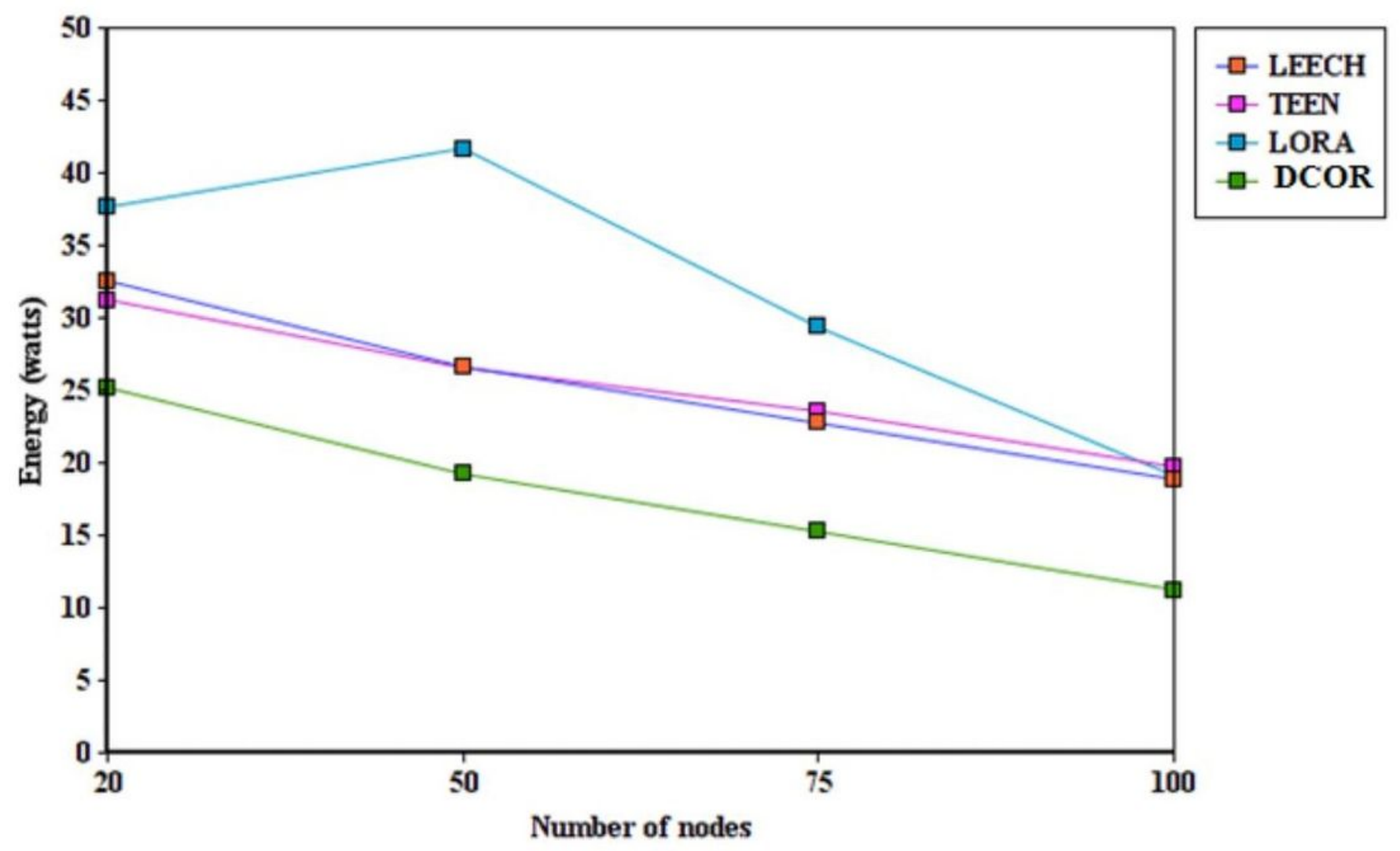

Figure 8

Energy Analysis of Proposed Approach 
Throughput analysis

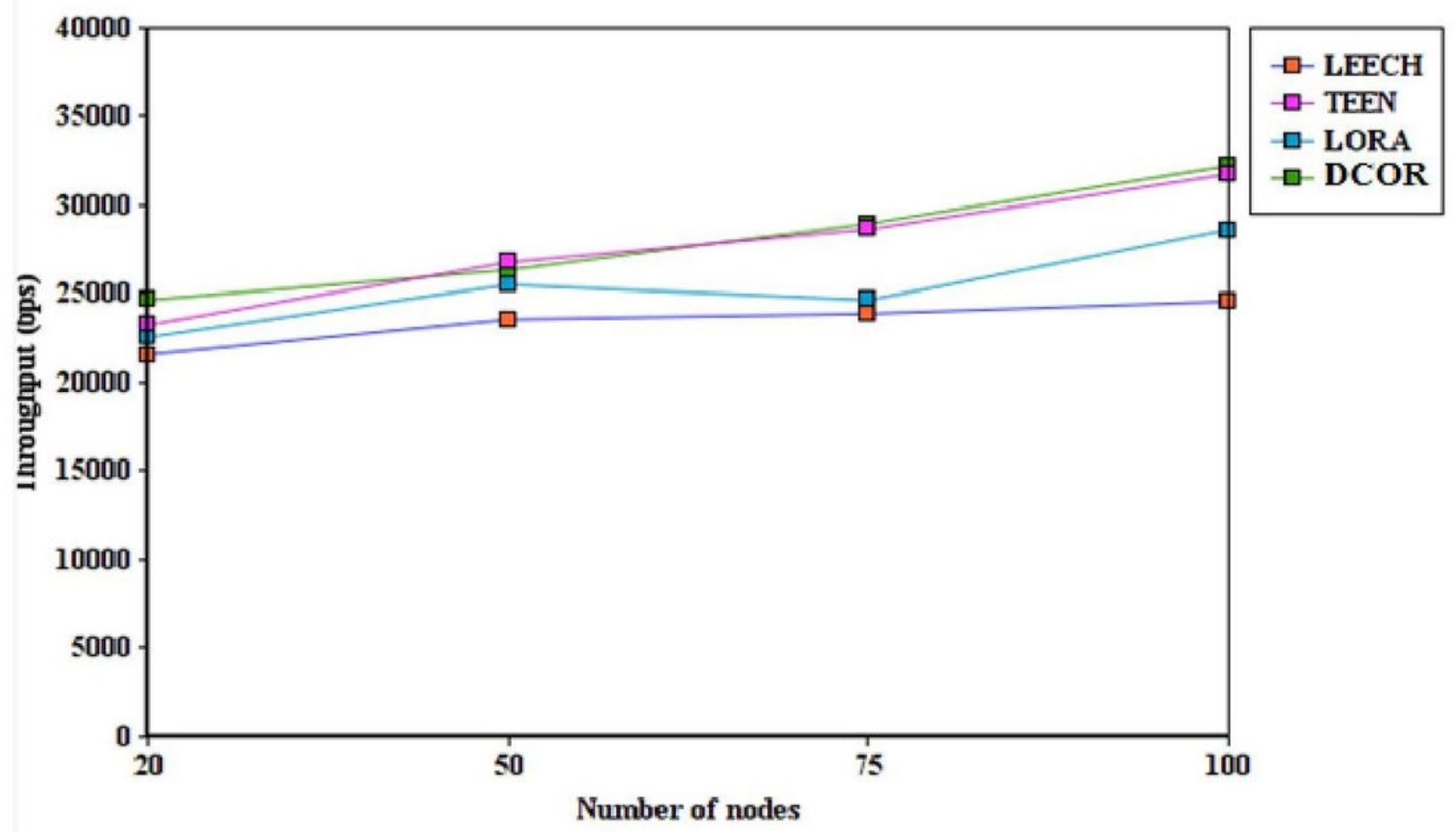

Figure 9

Throughput Analysis of DCOR 
Packet Delivery Ratio

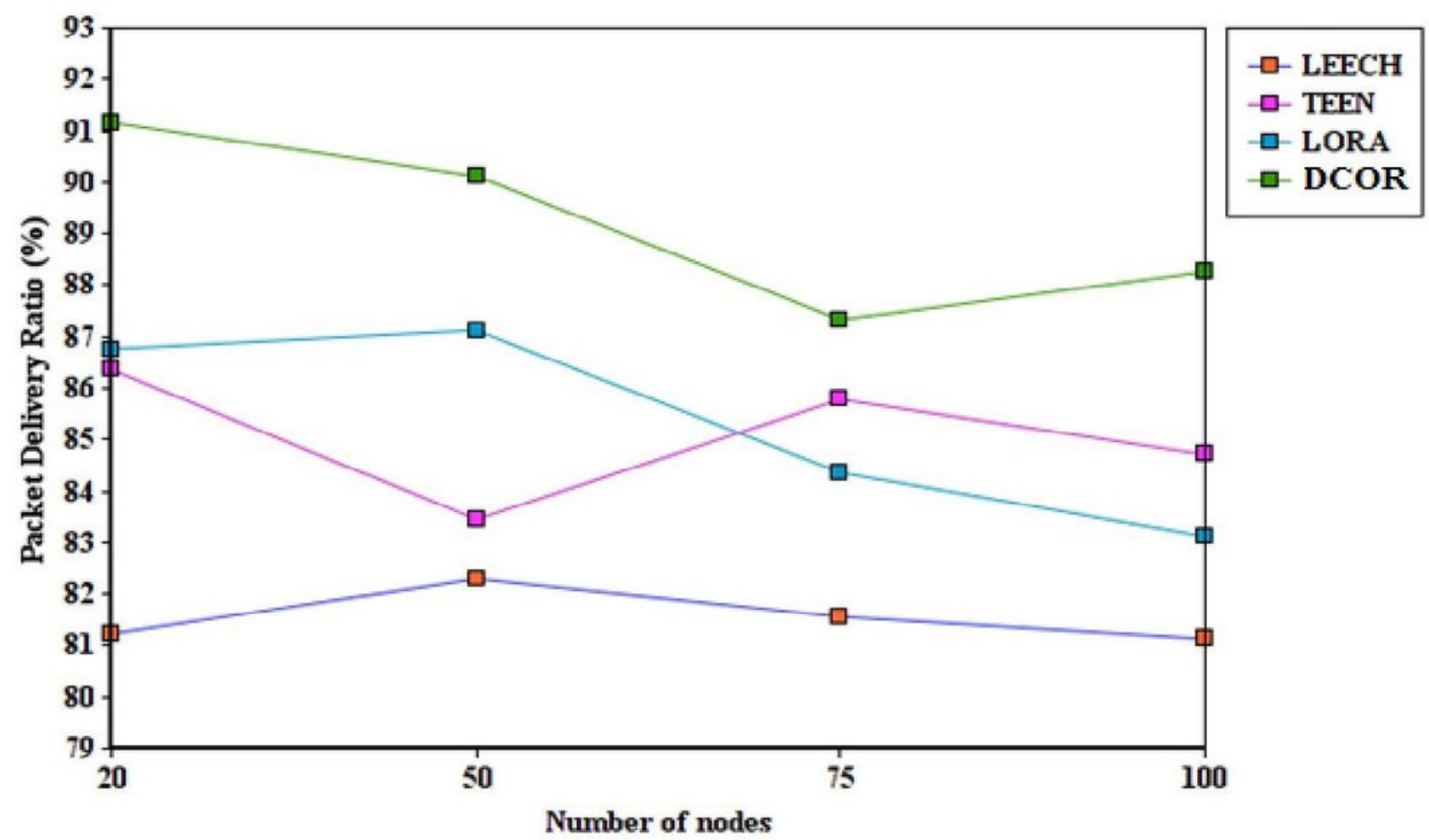

Figure 10

Packet Delivery Ratio of DCOR 
End To End Delay

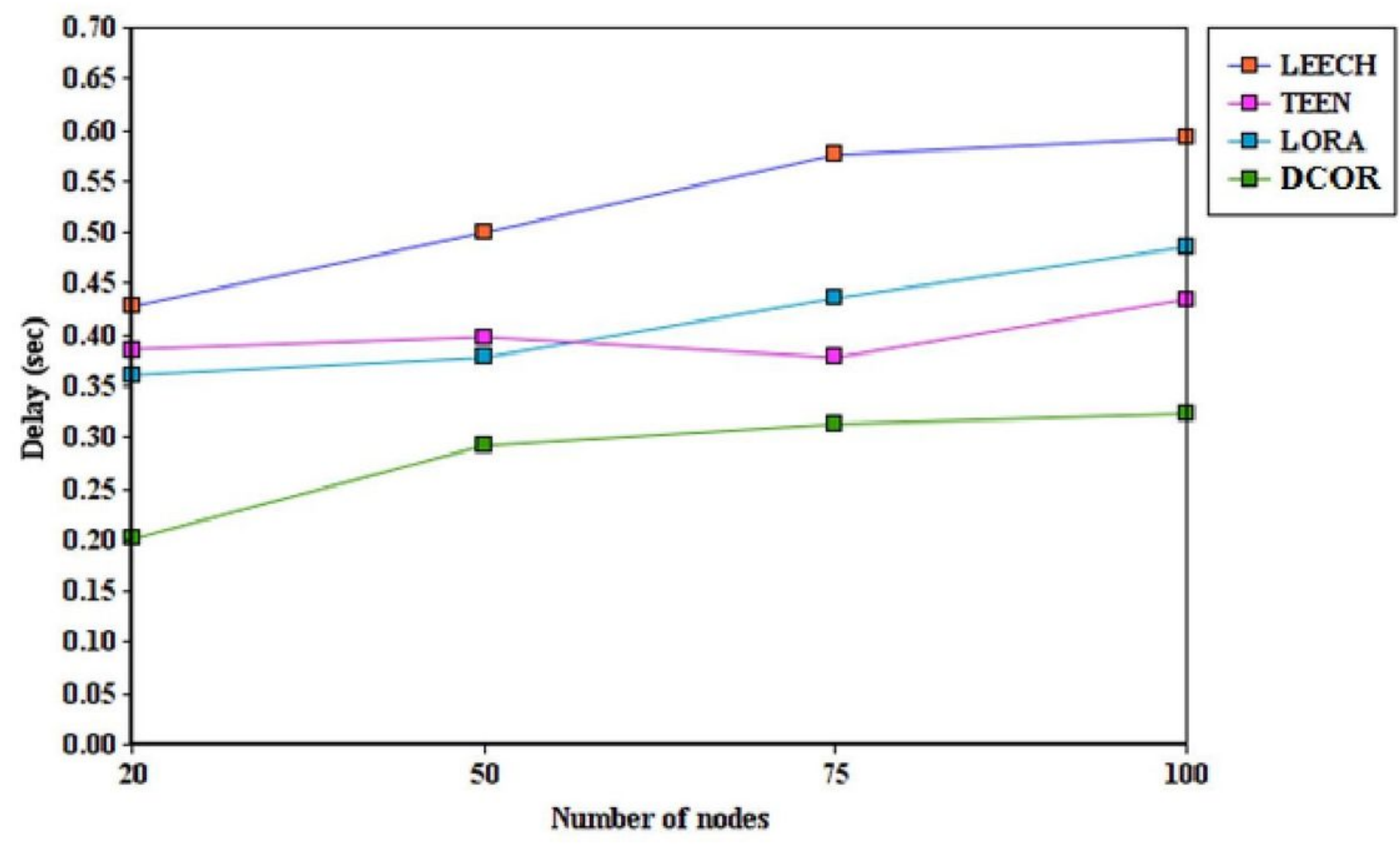

Figure 11

End to End Delay of DCOR 